\title{
Diversité Floristique Et Usages Des Ligneux Le Long Des Couloirs De Transhumance Dans La Commune De Savè Au Centre Bénin
}

\author{
Loukmane Moussa \\ Doctorant à l'Université d'Abomey-Calavi \\ Ibouraïma Yabi \\ Ismaïla Toko Imorou
}

Enseignant-Chercheur, Maître de Conférences, Université d'Abomey-Calavi

doi: 10.19044/esj.2016.v13n2p400 URL:http://dx.doi.org/10.19044/esj.2016.v13n2p400

\begin{abstract}
The vegetation cover of the District of Savè is subject to continuous pressure linked inter alia to the cutting of wood, agriculture and livestock through transhumance. Thus, forage seedlings are regularly used mainly during the dry season by transhumant herds.

In this context, the characterization of woody pastures and the study of the uses of its timber by the local populations is a major imperative for their preservation and sustainable management.

To this end, socio-economic surveys were carried out among the stakeholders involved in the use of these plant resources. The phytosociological surveys (29 plots of $30 \mathrm{~m} * 30 \mathrm{~m}$ ) were carried out using the sigmatite method of Braum blanquet. Different indices have been calculated. The graph of the raw and weighted spectra was carried out.

The characterization of the structure of the pasture shows a predominance of the individuals of circumference of between 30 and $50 \mathrm{~cm}$. Mesophanerophytes are the most abundant and dominant forms of life, while chronologically Sudanese and Sudano-Zambeze species are the most represented. The most represented families are the combretaceae the leguminoseae. The Shannon diversity index varies from 3.21 to 3.95 bits and the Piélou equitability from 0.72 to 0.83 from one pasture to another. Three (3) types of pasture were identified along transhumance corridors. Namely: the pasture at Lannea velutina and Bridelia ferruginea; Pasture in Trichilia emetica and Annona senegalensis and grazing in Pteleopsis suberosa and Pericopsis laxiflora. This study shows that the environmental conditions favor the installation of several species along the transhumance corridors. The uses of forage resources, such as pruning for feeding livestock, charcoal
\end{abstract}


making, fuelwood research, particularly in transhumance corridors, are not likely to Conservations.

Keywords: Woody fodder; Transhumance corridors; Use, Savè

\section{Résumé}

Le couvert végétal de la commune de Savè fait l’objet de pressions permanentes liées entre autres aux coupes de bois, à l'agriculture et à l'élevage à travers la transhumance. Dans ce contexte de pression anthropique croissante, l'évaluation de la diversité floristique et la connaissance des différentes utilisations des ligneux s'avère un impératif majeur pour permettre la préservation et la gestion durable de ces ressources. Pour ce faire, des enquêtes socio-économiques ont été menées auprès des acteurs concernés par l'utilisation de ces ressources végétales. Il s’agit des éleveurs, des agriculteurs et des charbonniers; au total 162 acteurs ont été enquêtés. Les relevés phytosociologiques ont été réalisés par la méthode sigmatiste de Braum blanquet. Au total, 29 placeaux de $30 \mathrm{~m}$ de côté ont été réalisés. Différents indices (de similitude de Jaccard ; de Shannon ; de diversité de Hill ; de diversité D de Simpson ; de diversité de Margalef ; et d’Equitabilité de Piélou) ont été calculés. Le diagramme des spectres bruts et pondérés ont été réalisés.

La caractérisation de la structure des pâturages montre une prédominance des individus de circonférence comprise entre 30 et $50 \mathrm{~cm}$. Les mésophanérophytes sont les formes de vie les plus abondantes et dominantes alors que sur le plan chronologique ce sont les espèces soudaniennes et les espèces soudano-zambéziennes qui sont les plus représentées. Les familles les plus représentées sont les combretaceae les léguminoseae. L’indice de diversité de Shannon varie de 3,21 à 3,95 bits et l'équitabilité de Piélou de 0,72 à 0,83 d'un pâturage à un autre. Trois (3) types de pâturage ont été identifiés le long des couloirs de transhumance: le pâturage à Lannea velutina et Bridelia ferruginea ; le pâturage à Trichilia emetica et Annona senegalensis et le pâturage à Pteleopsis suberosa et Pericopsis laxiflora. Les utilisations faites des ressources ligneuses fourragères sont: l'émondage pour nourrir le bétail, la fabrication du charbon de bois, la recherche du bois de feu. Ces formes d'usage ne sont pas favorables à la conservation de la biodiversité le long des couloirs de transhumance.

Mots clés : ligneux fourragers, diversité, couloirs de transhumance, utilisations, Savè. 


\section{Introduction}

L’élevage extensif reste une clé de voûte des activités des populations rurales en Afrique sub-saharienne par ses fonctions économique, écologique (transfert de matière organique) et sociale (Chirat et al., 2007). La transhumance transfrontalière a pris de l'importance au lendemain des grandes sécheresses des années soixante-dix et quatre-vingt (Tamou, 2002). Les pays sahéliens comme le Niger et le Burkina- Faso représentent alors les zones de départ tandis que les pays côtiers tels que le Bénin et le Togo sont considérés comme des zones d'accueil. La Commune de Savè au Bénin est l'une des zones d'accueil des transhumants (Saliou, 2015). La demande des ligneux fourragers surtouts en saison sèche est plus forte. Les feuilles et parfois les fruits de ces ligneux, constituent une part importante de la ration du bétail en saison sèche. Un discours récurent attribue à l'élevage la responsabilité de la dégradation d’écosystèmes (Hervé, 2008). Dans le secteur d’étude, la pression sur les ligneux en générale et ceux fourragers en particulier le long des couloirs de transhumance est de plusieurs ordres: supplémenter l'alimentation animale en période critique, servir de matière première pour la médecine traditionnelle, fournir du bois, des aliments pour l'homme, et des produits domestiques divers. Ainsi, l'homme par ses pratiques locales d'utilisation des terres, provoque une transformation des écosystèmes qui augmente au rythme de la croissance démographique (Ouédraogo et al., 2006).

En général, les phénomènes climatiques et anthropiques de la dégradation des ressources végétales ont été abondamment décrits dans la littérature, il existe cependant très peu d’études détaillées au niveau local sur la diversité floristique et l'usage fait des ligneux le long des couloirs de transhumance, dans la Commune de Savè (centre Bénin).

D’une manière générale, l'impact écologique de l'exploitation des ressources forestières est non seulement fonction de la composition floristique des formations végétales, de la nature et de l'intensité de la récolte des produits (Peters, 1997), mais aussi des facteurs sociaux et économiques (Betti, 2001).

L'environnement local du secteur d'étude est soumis non seulement à une pression anthropique croissante (colons agricoles) mais aussi à l'envahissement régulier des transhumants des pays de la sous-région tels que le Burkina-Faso, le Niger et à des variations climatiques.

Dans la perspective d'un développement local axé sur la lutte contre la pauvreté, la connaissance des espèces floristiques et des usages des ligneux le long des couloirs de transhumance, représente une étape importante pour intégrer les besoins des populations dans les prises de décision concernant la gestion durable des ressources végétales. La performance des stratégies à mettre en place pour réduire ou freiner la perte 
des espèces végétales est étroitement liée à la fiabilité des outils de leur mise en œuvre. Cette recherche est axé sur les ligneux, qui constituent les composantes permanentes des savanes. Elle vise à appréhender l'utilisation locale des ligneux fourragers le long des couloirs de transhumance d'une part et à analyser la diversité floristique des ligneux le long desdits couloirs d'autre part.

\section{Matériel et méthodes}

\section{1. Milieu d'étude}

La recherche a été conduite dans la Commune de Savè (Figure 1) située dans la zone de transition soudano-guinéenne au Bénin (Saliou, 2015). Elle est située entre $2^{\circ} 10^{\prime}$ et $2^{\circ} 48^{\prime}$ de longitude est et entre $7^{\circ} 42^{\prime}$ et $8^{\circ} 45^{\prime}$ de latitude nord.

On note deux grandes saisons : une saison humide qui s'étale de la $2^{\text {ème }}$ décade d'avril à fin septembre et une saison sèche qui s'étend de mioctobre à début mars. Les températures maximales varient en moyenne entre $29^{\circ} \mathrm{C}$ en août et $36,7^{\circ} \mathrm{C}$ en février. Cette région est l'une des plus arrosées du pays. La moyenne interannuelle des précipitations est de $1100 \mathrm{~mm}$ sur une période de 30 ans $(1980$ - 2010).

Les formations végétales notées sont les forêts galeries, les forêts claires et savanes boisées, les savanes arborées et arbustives les mosaïques de cultures et de jachères et les savanes saxicoles (Djojouwin, 2001). La Commune de Savè compte 87177 habitants en 2013 (INSAE, 2013) ce qui fait d'elle, l'une des communes les moins peuplées du pays. Les principales activités sont le commerce, l'agriculture et l'artisanat. 


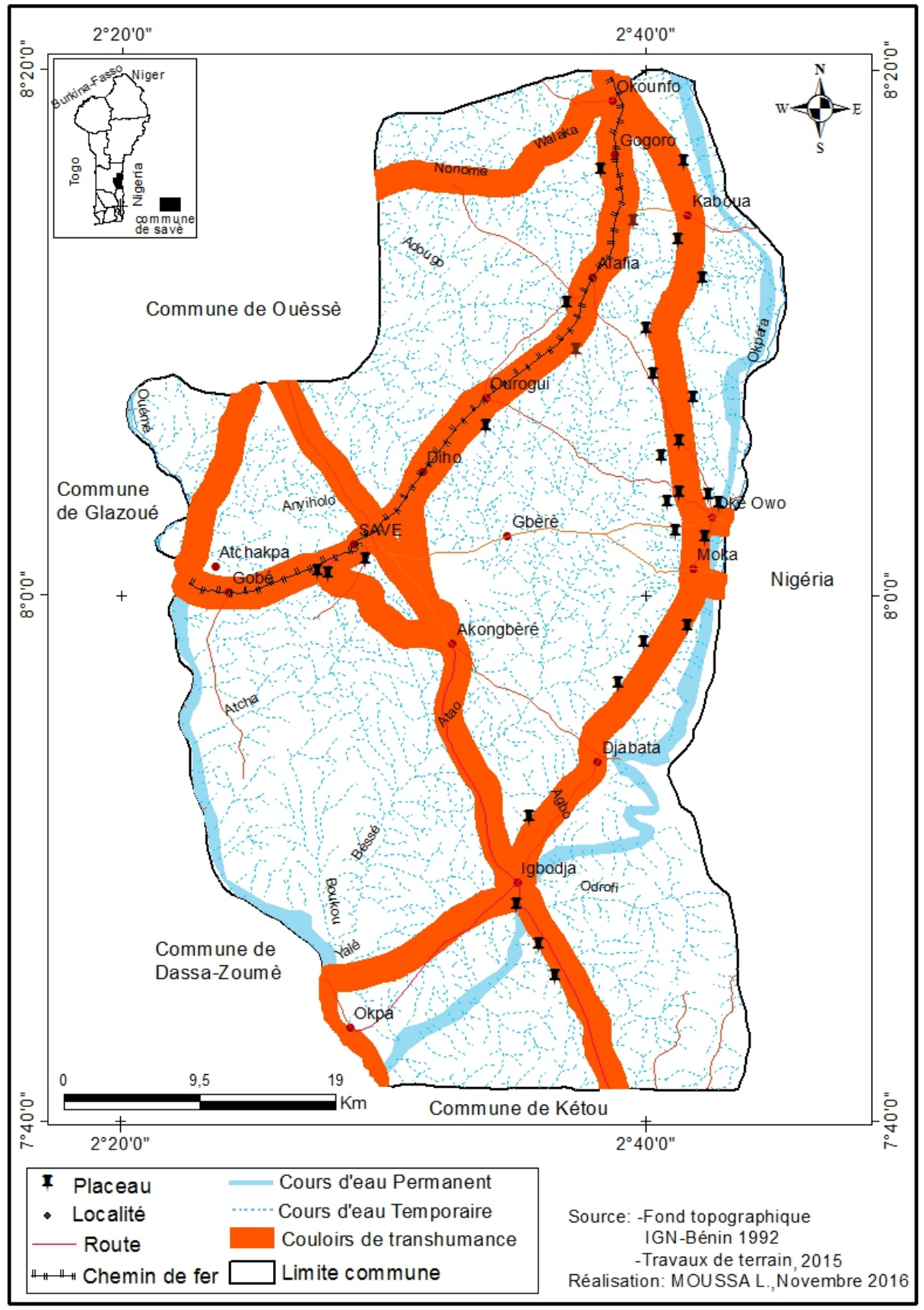

Figure 1 : Situation géographique des couloirs de transhumance dans le secteur d'étude

\subsection{Collecte des données}

\subsubsection{Collecte des données floristiques}

Au total, 29 placeaux ont été installés le long des couloirs de transhumance dans le secteur d'étude. L'un des critères de choix des 
placeaux est la présence des espèces ligneuses identifiées au préalable lors des enquêtes ethnobotaniques. L'aire des relevés de l'inventaire floristique retenue est basée sur les travaux effectués par plusieurs auteurs en milieu tropical selon les formations végétales et les strates. Ainsi l'aire de relevé est de $900 \mathrm{~m}^{2}(30 \mathrm{~m} \times 30 \mathrm{~m})$ adopté par (Agognon, 2004). Les relevés phytosociologiques ont été réalisés suivant la méthode sigmatiste de BraunBlanquet (1932) utilisée avec succès par plusieurs auteurs (Oumorou, 2003 ; Wala, 2004; Djego, 2006). Cette méthode est basée sur le principe d'homogénéité floristique de la surface étudiée. Les paramètres ou caractéristiques d'identification du site et les conditions stationnelles ont été notées. Il s’agit de :

$\checkmark$ recouvrement moyen de la strate arborescente;

$\checkmark$ type de formation végétale ;

$\checkmark$ type de sol ;

$\checkmark$ situation topographique.

Les informations relatives aux différents usages des ligneux ont été obtenues à partir des interviews directes. La technique d'enquêtes individuelles a été utilisée. L’âge des enquêtés varie entre 20 et 60 ans.

\subsubsection{Collecte des données socio-économiques}

Les principaux acteurs enquêtés sont ceux dont les activités touchent d'une manière ou d'une autre la végétation. Il s'agit essentiellement des agriculteurs et/ou tradipraticiens, des éleveurs, et des charbonniers. Les données statistiques sur l'effectif de ces différents acteurs n'étant pas disponibles, la taille de l'échantillon à enquêter au niveau de chaque catégorie d'acteurs a été déterminée à partir de la formule suivante (Dagnelie, 1998) adopté par Arouna (2012).

$$
\mathrm{n}=4 \mathbf{P}(\mathbf{1 - P}) / \mathbf{d}^{2}
$$

Avec : $\mathrm{P}=$ proportion de chaque catégorie d'acteurs obtenue à partir d'une enquête exploratoire, $\mathrm{d}=10 \%$ (marge d'erreur variant de 0 à $20 \%$ ). Au total, 162 individus ont été enquêtés au niveau des deux secteurs en tenant compte des localités où les couloirs de transhumance sont balisés (SCB) ou non balisés (SCNB), soit 81 acteurs par secteur (tableau I).

Tableaux I. Répartition et effectifs des différents acteurs enquêtés

\begin{tabular}{lcc}
\hline Acteurs & SCB & SCNB \\
\hline Eleveurs & 25 & 25 \\
Agriculteurs & 31 & 31 \\
Charbonniers & 15 & 15 \\
Exploitants forestiers & 10 & 10 \\
Total & 81 & 81 \\
\hline
\end{tabular}

Les observations directes ont été faites sur la base d'une grille d'observation, les entretiens individuels à l'aide d'un questionnaire 
spécifique à chaque catégorie socio-professionnelle, et 7 focus group regroupant en moyenne 8 personnes ont été réalisés.

\subsection{Traitement des données}

\subsubsection{Traitement des données floristiques}

L’individualisation des types de pâturage a été réalisée avec le logiciel CAP (Community Analysis Package). Le dendrogramme obtenu a permis d'individualiser les types de pâturages.

La détermination des espèces caractéristiques des pâturages individualisés a été faite par le logiciel PC-ORD 5.0. Les espèces caractéristiques d'un pâturage sont les espèces qui ont les plus fortes valeurs indicatrices et dont la fréquence relative dans les autres pâturages est $\leq 10 \%$ (pour les espèces présentes dans plus de deux pâturages) ou $\leq 15 \%$ (pour les espèces présentes seulement dans deux pâturages) (Toko, 2008).

Par ailleurs, les indicateurs de diversité spécifique des pâturages comme la richesse spécifique, l’indice de diversité de Shannon, l'équitabilité de Pielou l'indice de diversité de Margalef et l'indice de diversité de Hill ont été calculés.

- La richesse spécifique $(\mathrm{R})$ est le nombre d'espèces végétales recensées par placeau. Cet indicateur est insuffisant pour mesurer la diversité spécifique car il ne permet pas de différencier des pâturages qui comporteraient un même nombre d'espèces mais avec des effectifs différents (Arouna, 2012).

- L’indice de diversité de Shannon $(\mathrm{H})$ exprime la diversité des espèces au sein des pâturages. Il est calculé à partir de la formule suivante : $\mathrm{H}=-\Sigma \mathrm{Pi} \log _{2} \mathrm{Pi}$ avec $: \mathrm{Pi}=($ ni/N) est la fréquence relative des individus de l'espèce (i), (ni) est le nombre d'individus de l'espèce (i), (N) est le nombre total d'individus recensés.

- L’indice d’équitabilité de Pielou dérive de l'indice de Shannon (H) et mesure le degré de réalisation de la diversité maximale (Hmax) qui est égale au log à base 2 du nombre de taxons. Sa valeur montre la situation de régularité de la répartition des effectifs des différentes espèces (Fayé, 2010). L’équitabilité de Pielou (E) est déterminé par la formule : $\mathrm{E}=\mathrm{H} / \log _{2} \mathrm{R} \mathrm{La}$ valeur de l'équitabilité varie entre 0 et 1 . Elle tend vers 0 si la quasi-totalité des effectifs correspond à une seule espèce du peuplement et vers 1 lorsque chacune des espèces est presque représentée par le même nombre d'individus ou le même recouvrement.

- Dans l’optique de corriger le biais dû au fait que les sites étudiés n’ont pas la même taille que l'indice de Margalef a été calculé à partir de la formule suivante :

$$
\mathrm{MRg}=\frac{(\mathrm{S}-1)}{\ln (N)}
$$


Avec : MRg : Indice de diversité de Margalef ; S : Nombre d'espèces recensées $\mathrm{N}$ : Nombre total d’individus ; ln : logarithme népérien.

- L'indice D de Simpson mesure la probabilité que deux individus sélectionnés au hasard appartiennent à la même espèce : $\mathrm{D}=\sum_{i=1}^{s} \frac{\mathrm{ni}(\mathrm{ni}-1)}{\mathrm{n}(\mathrm{n}-1)}$; avec : ni le nombre d'individus de l'espèce $\mathrm{i}$ et $\mathrm{n}$ le nombre total d'individus inventoriés dans les placeaux. Cet indice varie entre 0 et 1 . Elle prend la valeur 0 pour indiquer le maximum de diversité et la valeur 1 pour indiquer le minimum de diversité.

- L’indice de diversité de Hill vient corriger les insuffisances probables de l'indice de Simpson. Il s'agit d'une mesure de l'abondance proportionnelle, permettant d'associer les indices de diversité de Shannon et de Simpson sa formule est : Hill $=\frac{1 / \mathrm{D}}{\operatorname{Exp}(\mathrm{H})}$; avec 1 / D l'inverse de l'indice de Simpson et Exp $(\mathrm{H})$ l'exponentiel de l'indice de Shannon de base 2. L'indice de diversité de Hill permet d'obtenir une vue encore plus précise de la diversité observée.

L'utilisation conjointe de ces indices a permis d'extraire un maximum d’informations sur les communautés végétales du milieu et de mieux comprendre leur niveau de diversité.

\subsubsection{Données structurales}

La densité (D) des ligneux est calculée selon la formule : $\mathrm{D}=\mathrm{N}$ x 10000/S avec D : nombre de tiges/ha ; $\mathrm{N}$ : nombre de tiges ayant au moins 2 $\mathrm{m}$ de hauteur ; $\mathrm{S}$ : superficie inventoriée rapportée à l’hectare. Les densités de la régénération par unité de surface.

Régénération $\mathrm{Re}=\mathrm{Nr} / \mathrm{S}$ avec $\mathrm{Nr}$ le nombre d’individus de la régénération et $\mathrm{S}$ : surface échantillonnée.

Surface terrière: la surface terrière moyenne est la somme des sections des troncs des arbres à un niveau de référence. Elle est calculée à partir de la formule suivante :

$$
\mathrm{Gi}=\Sigma \mathrm{C} 2 \times 10000 / 4 \Pi \mathrm{S}
$$

$\mathrm{C}$ : est la circonférence à $1,30 \mathrm{~m}$ du sol (m) ; S est la superficie inventoriée rapportée à l'hectare ; Gi est en $\mathrm{m}^{2} / \mathrm{ha}$.

\section{Répartition par classes de diamètre}

Le tableur Excel et le logiciel Minitab ont été utilisés respectivement pour la répartition par classes de diamètre et pour la réalisation de l'ajustement de Weibull. Pour mieux interpréter les structures en diamètre des pâturages, la distribution de Weibull à trois paramètres a été choisie. La distribution de Weibull à 3 paramètres ( $\mathrm{a}$, b et c) a pour fonction de densité de probabilité (Jonhson et Kotz, 1970) : $\mathrm{f}(\mathrm{x})=\frac{c}{b}\left(\frac{x-a}{b}\right)^{c-1} \exp \left[-\left(\frac{x-a}{b}\right)^{c}\right]$ 
Où $\mathrm{x}=$ diamètre des arbres; $\mathrm{f}(\mathrm{x})$ = valeur de densité de probabilité au point $\mathrm{x}$; $\mathrm{a}$ = paramètre d'origine (ou de position), il est égal à 0 si toutes les catégories d’arbres sont considérées (des plantules jusqu'aux semenciers), il est non nul si les arbres considérés ont un diamètre supérieur ou égal à a ; b = paramètre d'échelle ou de taille; il est lié à la valeur centrale des diamètres des arbres du peuplement considéré ; c = paramètre de forme lié à la structure en diamètre considérée. Les différentes caractéristiques de la végétation le long des couloirs de transhumance ainsi identifié permettent à la population locale différents usages.

\subsubsection{Traitement des données socio-économiques}

Les différents usages faits des ligneux dans le secteur d'étude ont été identifiés lors des travaux de terrain par l'observation directe sur la base d'une grille d'observation et des questions administrées aux riverains sur la base des questionnaires selon chaque catégories socio-professionnelles (Eleveurs, Agriculteurs et charbonniers).

\section{Résultats}

Les inventaires floristiques effectués le long des couloirs de transhumance ont permis de recenser 35 espèces ligneuses réparties en 36 genres et 18 familles. Les résultats de l'analyse de ces espèces ont permis de distinguer à $80 \%$ de dissemblance trois (3) grands types de pâturages. Il s’agit de: (1) pâturages à Lannea velutina et Bridelia ferruginea; (2) pâturage à Trichilia emetica et Annona senegalensis et (3) pâturage à Pteleopsis suberosa et Pericopsis laxiflora.

\subsection{Pâturage à Lannea velutina et Bridelia ferruginea}

\section{a. 2.1.1 Composition floristique et diversité spécifique}

b. 2.1.2 Spectres biologiques et phytogéographiques

La figure 2 présente les spectres des types biologiques et phytogéographiques du pâturage à Lannea velutina et Bridelia ferruginea.
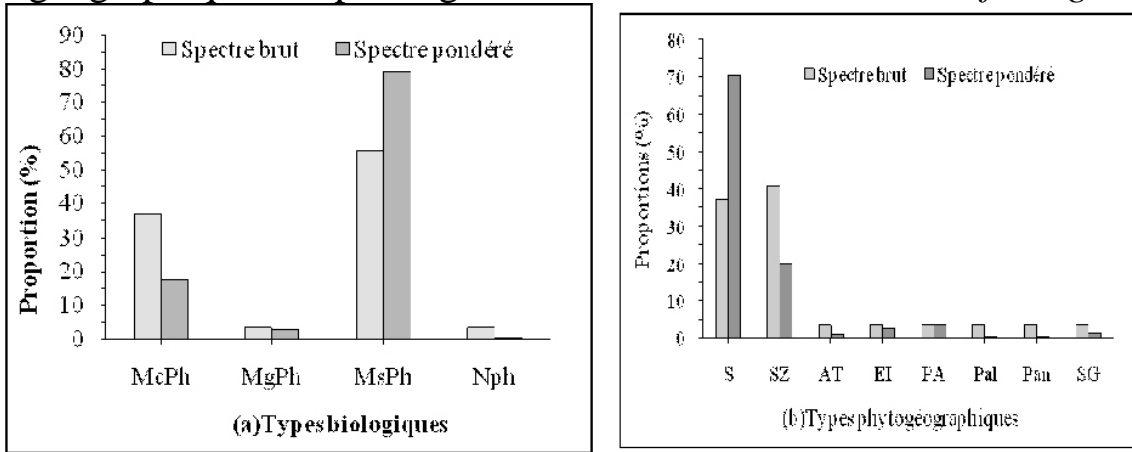

Figure 2 : Spectres des types biologiques (a) et phytogéographiques (b) du pâturage à Lannea velutina et Bridelia ferruginea. 
L'examen des types biologiques (figure 2a) révèle que les mésophanérophytes sont les formes de vie les plus abondantes et dominantes (79,13\% ; 55,56 \%) suivies des microphanérophytes (37,04 \%, 17,53\%). Les mégaphanérophytes et les nanophanérophytes ont un faible recouvrement au sol soit respectivement (3,70\%;2,93\%), et (3,70\%; 0,29 $\%)$. L’analyse des types phytogéographiques (figure $2 b$ ) révèle que les espèces de l'élément base soudanien (s) sont abondantes et dominantes (37,03\%; 70,35\%), ensuite viennent les espèces soudano-Zambéziennes $(40,74 \%$; 20,07 \%). Les autres formes de vie sont faiblement représentées.

\subsubsection{Caractéristiques structurales}

\subsubsection{Structure par classes de circonférence}

La figure 3 présente la structure par classes de circonférence du pâturage à Lannea velutina et Bridelia ferruginea des savanes arborées et arbustives. La structure des arbres de ce pâturage présente une distribution asymétrique positive. Avec un paramètre de forme " $\mathrm{c}$ » de la distribution de Weibull de l'ordre de 1,16. Ceci est caractéristique d'un peuplement monospécifiques avec prédominance d’individus jeunes.

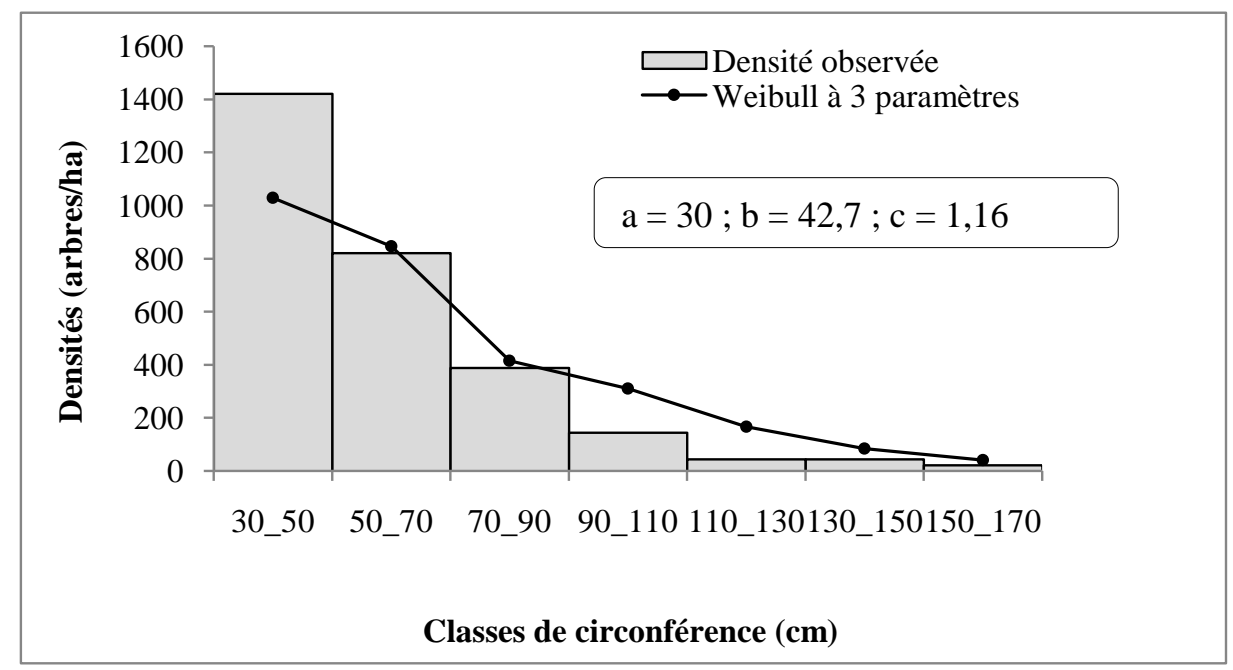

Figure 3 : Structure par classes de circonférence des arbres du pâturage à Lannea velutina et Bridelia ferruginea

Il ressort de l'analyse de la figure 2 que les individus de circonférences comprises entre $30 \mathrm{~cm}$ et $50 \mathrm{~cm}$ sont les plus abondants. Les espèces les plus rencontrées dans cette tranche de circonférence sont Anogeissus leiocarpa, Pterocarpus erinaceus et Vitellaria paradoxa. Ces espèces sont suivies respectivement de celles dont les circonférences varient entre $50 \mathrm{~cm}$ et $70 \mathrm{~cm}$ et sont constituées de Anogeissus leiocarpa, Pterocarpus erinaceus. Les autres classes de circonférences sont en de très 
faibles proportions. Les individus dont les circonférences varient entre 130 $\mathrm{cm}$ et $170 \mathrm{~cm}$ sont quasi inexistants au niveau des couloirs de transhumance.

\subsubsection{Densité et surface terrière}

La densité moyenne des individus de circonférence $\mathrm{C} \geq 30 \mathrm{~cm} \mathrm{du}$ pâturage est de 262,63 \pm 63,10 individus/ha. La surface terrière est de 6,47 \pm $2,41 \mathrm{~m}^{2} / \mathrm{ha}$. La faible valeur de la surface terrière confirme que les individus de grosses circonférences sont peu représentés.

\subsection{Pâturage à Trichilia emetica et Annona senegalensis}

c. 2.2.1 Composition floristique et diversité spécifique

d. 2.2.2 Spectres biologiques et phytogéographiques

La figure 3 présente les spectres biologiques et phytogéographiques du pâturage à Trichilia emetica et Annona senegalensis.
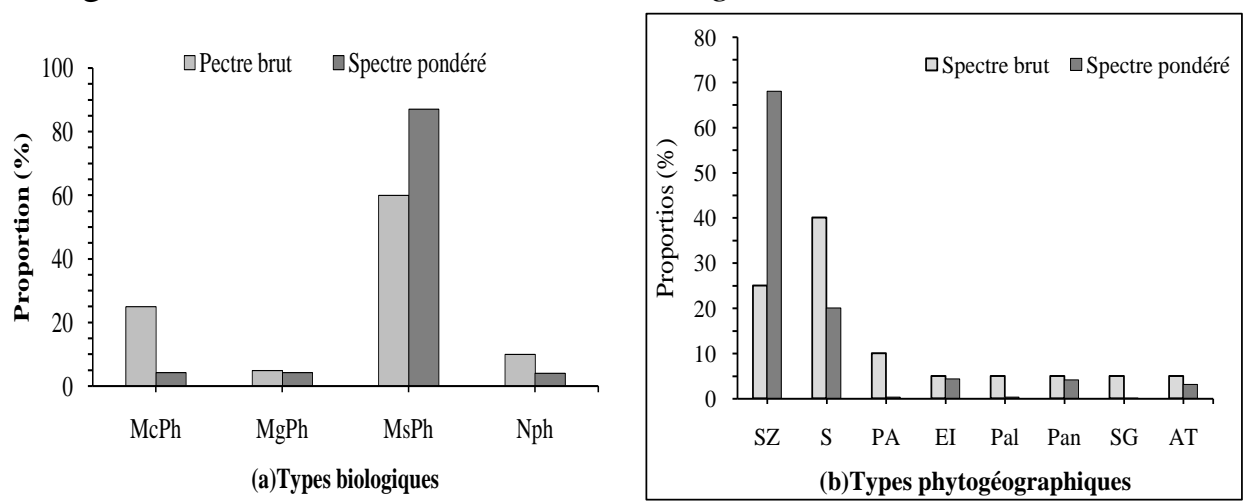

Figure 3 : Spectres biologiques et phytogéographiques du pâturage à Trichilia emetica et Annona senegalensis

L’examen de la figure 3a révèle que les mésophanérophytes (SB : $87,12 \%$; SP : $60 \%$ ) suivies des microphanérophites (SB : 25\% ; SP : 4,37 $\%)$ sont les formes de vie les plus abondantes et dominantes. Les nanophanérophytes (SB : $10 \%$; SP : 4,15 \%) viennent en 3ème position. Les mégaphanérophites sont en faible proportion.

L'analyse des types phytogéographiques (figure 3b) révèle que les espèces soudano- zambéziennes (sz) sont les plus abondantes et dominantes (25 \% ; 67,85 \%), ensuite viennent les espèces soudaniennes (40\%; 19,98 $\%)$ et les espèces plurirégionales africaines (10\%;0,25\%). Les autres formes de vie sont faiblement représentées.

\subsubsection{Caractéristiques structurales}

\subsubsection{Structure par classes de circonférence}

La figure 4 présente la structure par classes de circonférence du pâturage à Trichilia emetica et Annona senegalensis des savanes arborées et 
arbustives. La structure des arbres de ce pâturage présente une distribution asymétrique positive. Avec un paramètre de forme « $\mathrm{c}$ » de la distribution de Weibull de l'ordre de 1,23. Ceci est caractéristique d'un peuplement monospécifiques avec prédominance d'individus jeunes.

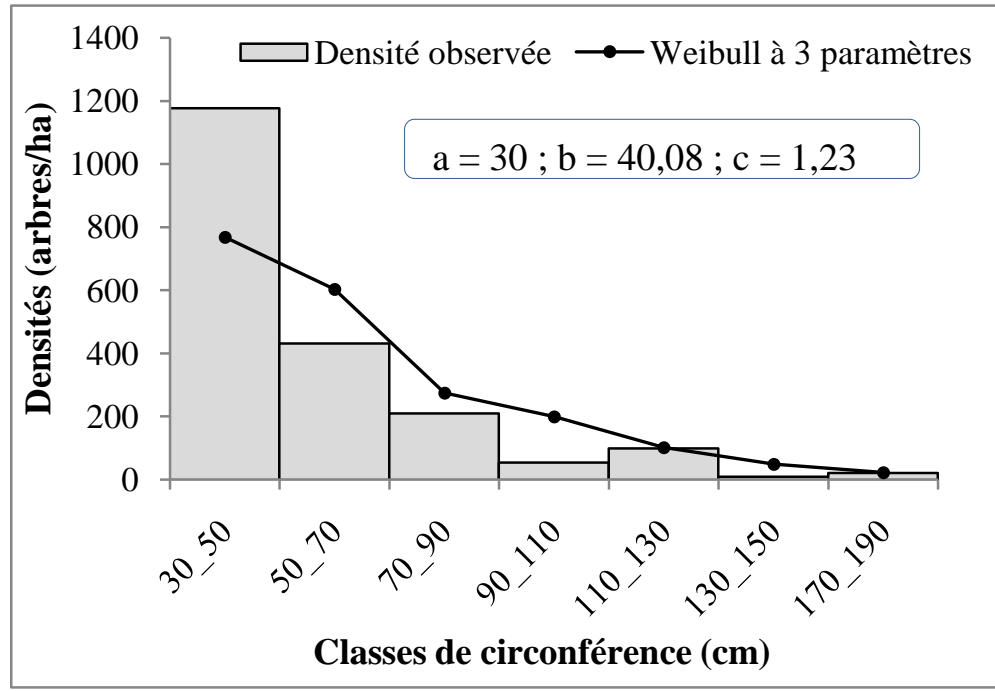

Figure 4 : Structure par classes de circonférence des arbres du pâturage à Trichilia emetica et Annona senegalensis

Il ressort de l'analyse de la figure que les individus de circonférences comprises entre $30 \mathrm{~cm}$ et $50 \mathrm{~cm}$ sont les plus abondants. Les espèces les plus rencontrées dans cette tranche de circonférence sont Daniellia oliveri, Parinari curatellifolia et Combretum collinum.

Ces espèces sont suivies de celles dont les circonférences varient entre $50 \mathrm{~cm}$ et $70 \mathrm{~cm}$ et sont constituées de Daniellia oliveri et Pterocarpus erinaceus. Les classes de circonférences comprises entre $70 \mathrm{~cm}$ et $90 \mathrm{~cm}$ sont en des proportions relativement faibles. Elles sont constituées des espèces telles que Daniellia oliveri et Bridelia ferruginea. Les individus dont les circonférences varient entre 90 à 190 sont en de faibles proportions.

\subsubsection{Densité et surface terrière}

La densité moyenne des individus de circonférence $\mathrm{C} \geq 30 \mathrm{~cm} \mathrm{du}$ pâturage est de $223 \pm 110$ individus/ha. La surface terrière est de 6,65 \pm 3,97 $\mathrm{m}^{2} / \mathrm{ha}$. La faible valeur de la surface terrière confirme que les individus de grosses circonférences sont peu représentés

\subsection{Pâturage à Pteleopsis suberosa et Pericopsis laxiflora}

Ce type de pâturage est présent dans les savanes arborées et arbustives et se rencontre au niveau de plusieurs facettes topographiques (bas de versant, mi- versant) sur des sols à texture 
limono-sableuse et sablo-graveleuse.

\subsubsection{Composition floristique et diversité spécifique}

Le pâturage à Pteleopsis suberosa et Pericopsis laxiflora est identifié sur la base de 9 relevés, Il est constitué de 22 espèces ligneuses réparties en 22 genres et en 12 familles.

Les familles les plus représentées sont: combretaceae (22,73\%), Leguminosae-Caesalpinoideae (18,18 \%), Leguminosae-Papilionoideae $(13,64 \%)$, leguminosae-mimosoideae $(9,09 \%)$. Les six premières espèces caractéristiques de ce pâturage sont: Pteleopsis suberosa, Pericopsis laxiflora, Prosopis africana, Combretum molle, Sterculia setigera et Holarrhena floribunda. La richesse spécifique varie de 3 à 10 espèces/placeau. L’indice de diversité de Shannon est de 3,21 \pm 3 bits avec une équitabilité de Pielou de 0,72 . On peut conclure que les conditions du milieu sont favorables à l'installation de nombreuses espèces, et que chacun des espèces est presque représenté par le même nombre d'individus.

\subsubsection{Spectres des types biologiques et des types phytogéographiques}

$\mathrm{La}$ figure 5 présente les spectres des types biologiques et phytogéographiques du pâturage à Pteleopsis suberosa et Pericopsis laxiflora.

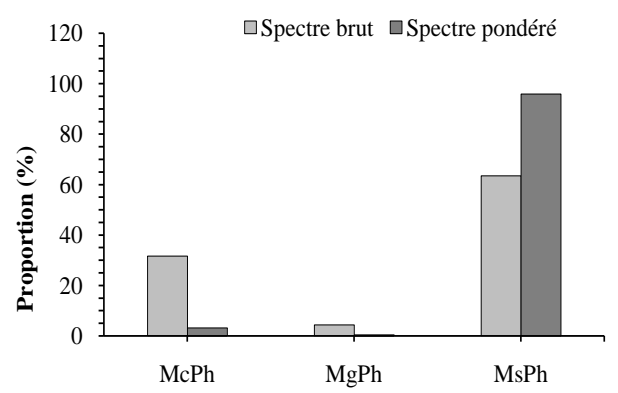

(a)Types biologiques

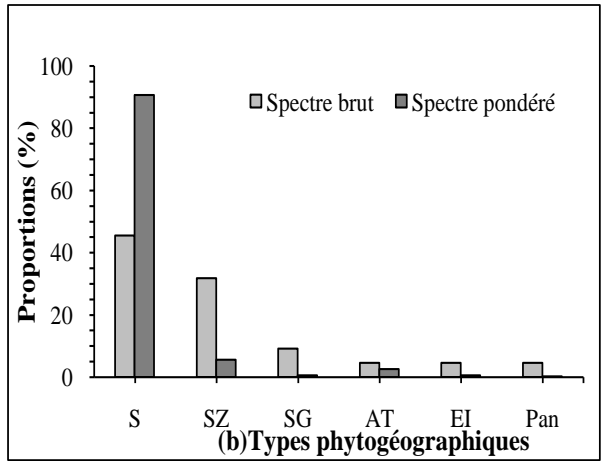

Figure 5 : Spectres biologiques et phytogéographiques du pâturage à Pteleopsis suberosa et Pericopsis laxiflora

L'examen de la figure $5 a$ révèle que les mésophanérophytes (SB : 63, $64 \%$; SP : 96,02 \%) suivies des microphanérophytes (SB : 31,82 \% ; SP : $3,40 \%$ ) sont les formes de vie les plus abondantes et dominantes. La faible proportion des mégaphanérophytes témoigne de la forte pression anthropique sur les espèces ligneuses le long des parcours de transhumance du secteur d'étude.

L'analyse des types phytogéographiques (figure $5 b$ ) révèle que les espèces soudaniennes $(45,45 \%$; 90,59 \%) sont les plus abondantes et 
dominantes. Elles sont suivies des espèces soudano-zambéziennes (31,82 \% et 5,62 \%). Les autres formes de vie sont faiblement représentées.

\subsubsection{Caractéristiques structurales}

\subsubsection{Structure par classes de circonférence}

La figure 6 présente la structure par classes de circonférence du pâturage à Pteleopsis suberosa et Pericopsis laxiflora des savanes arborées et arbustives. La structure de ce pâturage a une distribution asymétrique droite, avec un paramètre de forme « $\mathrm{c}$ » de la distribution de Weibull de l'ordre de 1,28. Ceci est caractéristique d'un peuplement mono-spécifiques avec prédominance d'individus jeunes.

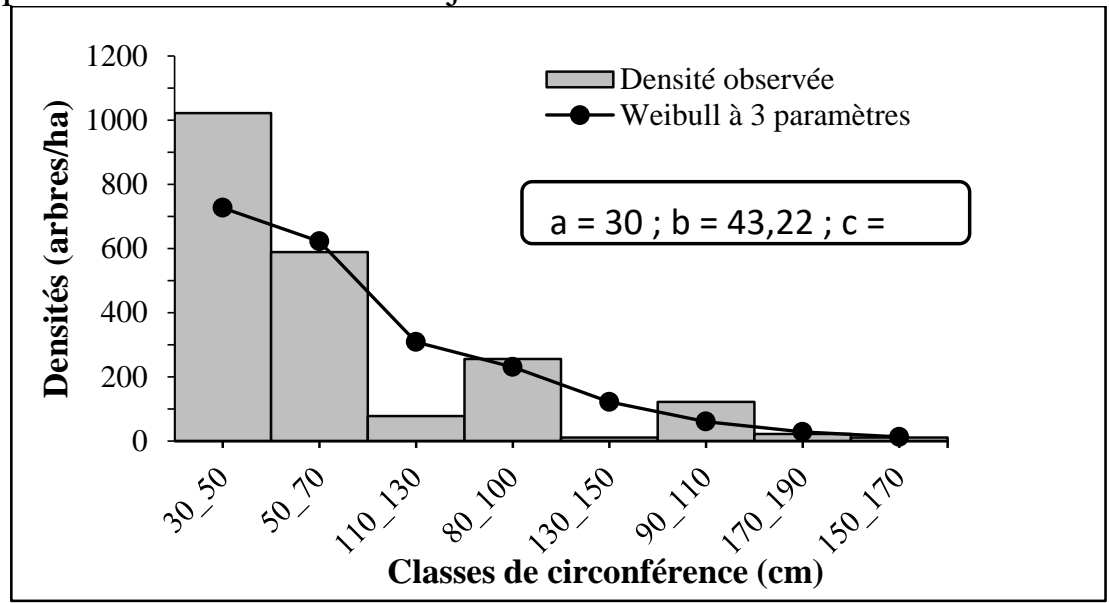

Figure 6 : Structure par classes de circonférences des arbres du pâturage à Pteleopsis suberosa et Pericopsis laxiflora

L'examen de la figure 6 montre que les individus de circonférences comprises entre $30 \mathrm{~cm}$ et $50 \mathrm{~cm}$ sont les plus abondants. Les espèces les plus rencontrées dans ces classes de circonférences sont Anogeissus leiocarpa, Pterocarpus erinaceus et vitellaria paradoxa. Ils sont suivis des individus dont les circonférences sont comprises entre $50 \mathrm{~cm}$ et $70 \mathrm{~cm}$, et entre $70 \mathrm{~cm}$ et $90 \mathrm{~cm}$. Les espèces les plus rencontrées dans cette frange sont respectivement Anogeissus leiocarpa et Pterocarpus erinaceus. Viennent ensuite les individus de circonférences comprises entre $90 \mathrm{~cm}$ et $110 \mathrm{~cm}$. Ils y sont présents mais en de très faibles proportions. Quant aux individus de circonférences supérieures à $110 \mathrm{~cm}$, ils sont quasi-absents du pâturage. Il s'agit notamment des individus de Vitellaria paradoxa, Pterocarpus erinaceus et Afzelia africana.

\subsubsection{Densité et surface terrière}

La densité moyenne des individus de circonférence $\mathrm{C} \geq 30 \mathrm{~cm} \mathrm{du}$ pâturage est de 234,57 \pm 
116,99 arbres/ha. La surface terrière est de 7,51 $\pm 3,52 \mathrm{~m}^{2} / \mathrm{ha}$. Cette faible valeur indique la faible représentativité des individus de grosses circonférences dans ce pâturage.

\subsection{Usages des ligneux le long des couloirs de transhumance}

Selon les différents besoins des populations du secteur d'étude, le prélèvement des ligneux se fait de plusieurs manières.

\subsubsection{Fabrication du charbon de bois}

La fabrication du charbon de bois dans le secteur d'étude se fait par coupe des arbres de toutes dimensions (du plus grand au plus petit). Les espèces les plus désirées pour la fabrication sont: Khaya senegalensis, Milicia excelsa et Pterocarpus erinaceus.

Le fabricant du charbon fait des tas de bois coupé, met le feu et par une combustion incomplète du tas, sort du charbon de bois prêt à être commercialisé. Cette forme d'utilisation des ligneux est fréquemment observée. Le bois est aussi brulé au niveau des foyers de circonstance, constitués par trois blocs de pierres supportant les marmites pour la cuisson des aliments.

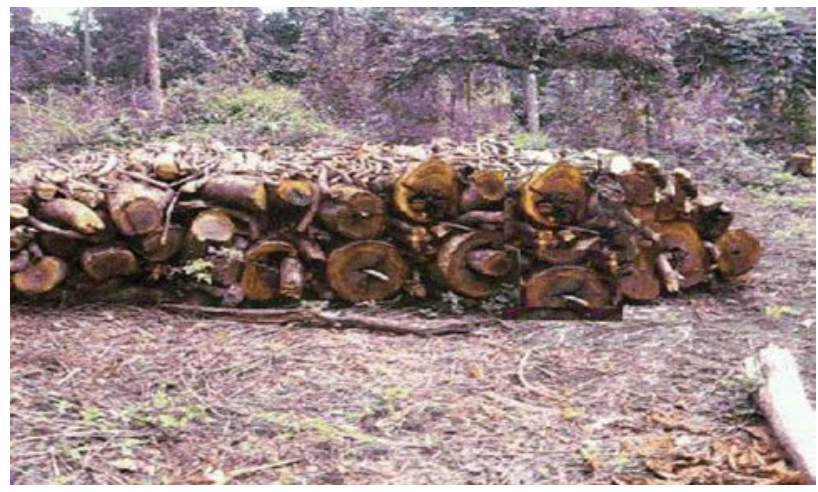

Photo 1 : Tas de bois de toutes dimensions apprêté pour la fabrication du charbon de bois

\subsubsection{Emondage des ligneux fourragers}

Chaque année le secteur d'étude accueille des éleveurs étrangers venant essentiellement du Burkina-Faso, du Niger, du Nigéria. Pour satisfaire leurs bétails en fourrages, les transhumants n'hésitent pas à couper les ligneux fourragers.

\subsubsection{Utilisation des ligneux dans la médecine traditionnelle}

Pour des raisons de médecine traditionnelle, les populations locales utilisent soit les feuilles, les écorces, les racines, et mêmes les fleurs des 
arbres telles que: Pterocarpus eurinaceus, Khaya senegalensis, Milicia excelsa, Zanthoxylum zanthoxyloïdes qui se révèlent très efficaces dans le traitement de certaines maladies. Ses différents prélèvements se font beaucoup plus sur les couloirs de transhumance car il est plus facile à un guérisseur traditionnel de suivre les couloirs qui sont moins envahis par les espèces dédaignées.

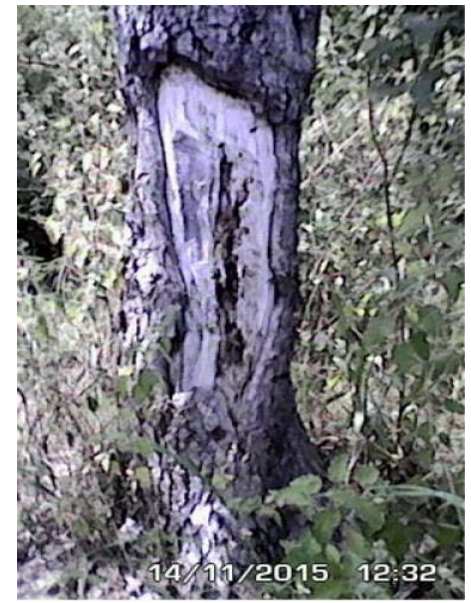

Photo 2 : Pterocarpus erinaceus écorcé à des fins médicinales

\subsubsection{Utilisation des ligneux dans la construction et l'artisanat}

Les ligneux constituent une source de matières premières pour la construction et l'artisanat. Les exploitants forestiers coupent systématiquement les arbres dont les diamètres sont suffisants pour en faire des bois d'œuvre. Les populations de Savè produisent aussi à partir du bois des écorces ou des feuilles des produits artisanaux aussi utiles que variés à savoir chaises, lits, cordes, nattes, éventails, frottoirs, paniers, pilons, mortiers, charpentes de maison.

\section{Discussion}

\subsection{Caractérisation des pâturages}

Les résultats de l'analyse multivariée des relevés phytosociologiques effectués le long des couloirs de transhumance des troupeaux transhumants dans la Commune de Savè ont permis d'identifier trois (3) types de pâturage : le pâturage à Lannea velutina et Bridellia ferruginea, le pâturage à Trichilia emetica et Annona senegalensis, le pâturage à Pteleopsis suberosa et Pericopsis laxiflora. En général l'interprétation des différents indices de végétation calculés montre que les conditions du milieu sont favorables à l'installation de nombreuses espèces. Ces résultats sont conformes à ceux de Toko Imorou (2014) qui a mené une étude au niveau des parcours naturels des troupeaux transhumants en zone Soudano-guinéenne au nord Bénin. Il a aussi abouti aux mêmes résultats et obtenu des indices de diversité de 
Shannon élevés (4,07 bits). D’autres études menées sur les systèmes d'utilisation des terres en général ont également eu des résultats similaires. Ainsi, respectivement au Sénégal et au Mali, Mbow et al. (2008) et Yossi (1996) après des études sur l’évaluation de la diversité ligneuse dans les systèmes d'utilisation des terres en zone soudanienne, en sont arrivés à conclure l'effet négatif de la pression anthropique sur la diversité ligneuse des formations végétales. Ainsi les résultats obtenus pour cette étude montrent que la dynamique constatée au niveau de la végétation dans le secteur d'étude dépend des actions anthropiques.

D’une manière générale, les trois types de pâturages de savanes étudiés sont caractérisés par l'abondance et la dominance des mésophanérophytes. La forte proportion des mésophanérophytes pourrait s'expliquer par la pression anthropique qui s’observe le long des couloirs de transhumance du secteur d'étude, caractérisée par l'abondance de jeunes plantes. Selon Sounon Bouko et al. (2007), on note au Bénin, la modification de la structure de la végétation, la baisse de la diversité spécifique et de la densité des ligneux de gros diamètres. La similarité est noter au niveau de la prédominance des ligneux de faibles diamètres, au détriment des ligneux de gros diamètres.

La richesse spécifique du secteur d’étude est de 35 espèces réparties en 36 genres et 18 familles obtenues dans 29 relevés. La caractérisation de la structure de la végétation des différents types de pâturage dans la commune d'étude a montré la prédominance des individus de circonférences comprises entre 30 et $50 \mathrm{~cm}$. Les familles les plus représentées sont les Combretaceae et les Leguminosae. Les résultats similaires ont été déjà obtenus en Côte d'Ivoire par les travaux de Charahabil et al., (2012) en zone de transition soudano-guinéenne, ce qui avait amené l'auteur à utiliser le terme de « combrétinisation » du milieu. On peut affirmer avec cet auteur que la famille des Combretaceae est celle qui semble de loin s'adapter le mieux aux conditions du secteur d’étude. Les mésophanérophytes sont les formes de vie les plus abondantes et dominantes alors que sur le plan chronologique, ce sont les espèces soudaniennes et les espèces soudano-zambéziennes qui sont les plus représentées.

Les spécificités phytogéographiques sont alors maintenues malgré la dynamique régressive de ces formations végétales. Cela confirme la relative stabilité du secteur d'étude sur le plan phytogéographique. La faible représentativité des mégaphanérophytes témoigne de la forte pression anthropique sur les ligneux de grands diamètres. En effet, la synthèse des résultats des quatre recensements généraux de la population et de l'habitation (INSAE, 2002 et INSAE, 2013) montrent que la population de la Commune de Savè est passée respectivement de 26117 à 87177 entre 1978 et 2013. Cette croissance rapide de la population a entraîné l'extension des surfaces 
cultivées et l'augmentation des besoins en bois, mais il faut aussi noter la coupe massive des ligneux de gros diamètre à des fins commerciales vers d’autres pays. Ce qui explique leur rareté au niveau de la végétation.

Aussi, les travaux de Arouna (2012), ont-ils montré que dans la Commune de Djida, l’émondage est également une pratique répandue et utilisée par tous les éleveurs pour nourrir leurs troupeaux, surtout en période de soudure. Les passages répétés des troupeaux transhumants nationaux et transfrontaliers engendrent une dégradation des sols et du couvert végétal. Les mêmes constats ont été faits par Tamou (2002) et Lessé (2011) qui ont montré que le couvert végétal s’éclaircit davantage, la biomasse diminue. Ces différents résultats obtenus par les chercheurs dans des localités différentes en zone soudano-guinéenne confirment les résultats obtenus dans le cadre de ce travail de recherche.

\subsection{Usages des ligneux}

Dans la commune de Savè, le bois reste la principale source de cuisson des aliments parce qu'il nécessite moins d’investissement relativement aux autres modes de cuissons. En effet, seulement, le bois est utilisé sans appareils de chauffage pour la cuisson. Alors que cette combustion du bois sans dispositif de contrôle des courants d'air consomme de plus grandes quantités de bûches et constitue en soi un problème pour l'environnement (Dongotou, 2010). La fabrication du charbon de bois dans le secteur d'étude est faite par la coupe systématique des ligneux de toutes tailles. Ce mode d'utilisation de ces ligneux laissent des espaces seulement herbeux par endroits. Les espèces telles que Vitellaria paradoxa (Karité) jadis interdite de coupe pour la fabrication du charbon de bois n'est plus respectée de nos jours. Aussi note-t-on l'utilisation des fruits de certains ligneux (après leurs transformations) dans la cuisine. C'est le cas de Parkia biglobosa (Néré) dont le fruit sert à fabriquer la moutarde locale très appréciée dans la cuisine. Aussi plusieurs usages sont-ils faits des différentes parties des arbres (écorces, racines, feuilles) pour des raisons de médecine traditionnelle.

Une étude menée au Sénégal par Gneing en 2013, sur la valeur socioéconomique de l'arbre en milieu malinké dans la localité de Khosonto au Sénégal a abouti aux résultats similaires à ceux obtenus dans le cadre de cette recherche dans la Commune de Savè au centre Bénin. Ainsi, Gneing et al. (2013) ont constaté qu'en plus de la consommation directe des fruits comestibles, les feuilles d'espèces ligneuses entrent dans plusieurs mets locaux.

Après celle de l'homme, l'alimentation du bétail constitue le domaine d'usage le plus important des ligneux. Cette importance des ligneux en tant que fourrage, tient au fait qu'ils apportent protéines, minéraux et vitamines 
indispensables à l’équilibre alimentaire du bétail, donc à la vie des troupeaux au cours des mois de saison sèche, et à leur survie lors de sécheresse anormale (Le Houérou, 1980). Dans la Commune de Savè, les ligneux servent de pâturage aux pasteurs transhumants en tant que fourrage en apportant des compléments alimentaires indispensables aux bétails en transit au Bénin. Parfois d’autres transhumants satisfaits du niveau de pâturage dans la Commune de Savè y restent pendant toute la saison critique dans leurs pays d'origine. Il ressort de ce travail de recherche sur les ligneux le long des couloirs de transhumance dans la Commune de Savè, que plusieurs usages sont faits des ligneux.

\section{Conclusion}

L’inventaire floristique le long des couloirs de transhumance dans la Commune de Savè a permis de recenser 36 espèces soit $1,17 \%$ de la flore du Bénin. La classification hiérarchique ascendante à l'aide du logiciel CAP (Community Analysis Package) des relevés phytosociologiques a permis d’identifier 3 pâturages. En général, les conditions du milieu sont favorables à l'installation de plusieurs espèces. La diversité spécifique est maximale au niveau de tous les pâturages. L'analyse des types biologiques révèle que les mésophanérophytes sont les formes de vie les plus abondantes et dominantes, suivies des microphanérophytes. La répartition des types phytogéographiques révèle que les espèces soudaniennes sont abondantes et dominantes ensuite viennent les espèces soudano-zambéziennes.

L’émondage, la fabrication du charbon de bois, les variabilités des pluies, l'accroissement du cheptel ont été perçus par les différentes catégories socio-professionnelles comme des facteurs de dégradation de la végétation le long des couloirs de transhumance dans la Commune de Savè.

\section{References:}

1. Agognon G. 2004. Rôle de l’agroforesterie dans la gestion intégrée des terroirs agropastoraux dans le département du Borgou. Th. Ir. Agr. FSA/UAC p 89.

2. Arouna O., 2002. L'exploitation des ressources biologiques et la dynamique de la forêt classée de l’Alibori supérieur (secteur de l'arrondissement de Bagou), Mémoire de maîtrise, UAC/FLASH/DGAT, 115 p.

3. Arouna O., 2012. Cartographie et modélisation prédictive des changements spatio-temporels de la végétation dans la Commune de Djidja au Benin : Implications pour l'aménagement du territoire. Thèse de doctorat en Géographie, UAC, 246 p.

4. Camou-Guerrero A., Reyes-García V., Martínez-Ramos M., et Casas A.; 2008 ' Knowledge and use value of plant species in a Rarámuri 
community: a gender perspective for conservation’, Human Ecology, vol. 36, pp 259-272.

5. hirat G., Ickowicz A., Bocquier F., 2007. Renc. Rech. Ruminants, 14, 191 pp. 327-330

6. Dagnelie P., 1998. Statistique théorique et appliquée (Tome 2). De Boeck \& Larcier, Paris- Bruxelles, 659 p.

7. Dongotou T., 2010. Repenser le développement durable au XXIe siècle, Éditions L'Harmattan, p. 66, 67.

8. Diallo H., Faye E. H., Koné B., Bindelle J., Lejoly J. et Maiga M., 2010. Boidiversité et valeurs pastorale des herbacées de la réserve de Fina (Mali) in African Plant Diversity Systematics and Sustainable Developpment. Proceedings of the XIXth : 111-120.

9. Djodjouwin L. 2001. Etude sur les aménagements écotouristiques et la gestion pastorale dans les terroirs des forêts classées des Monts Kouffé et de Wari Maro.Université d'AbomeyCalavi, Cotonou. Mémoire de DESS, 98 pages + annexes.

10. Diallo H., Faye E. H., Koné B., Bindelle J., Lejoly J. et Maiga M., 2010. Boidiversité et valeurs pastorale des herbacées de la réserve de Fina (Mali) in African Plant Diversity Systematics and Sustainable Developpment. Procedings of the XIXth : 111-120.

11. INSAE, 2002 : troisième Recensement général de la population et de l'habitat : Synthèse des analyses en bref $48 \mathrm{p}$.

12. INSAE, 2013 : quatrième recensement général de la population et de l'habitat : résultat définitif 33p.

13. Lessé P., 2011. Analyse de la gestion pastorale et de l'adaptation des eleveurs transhumants face aux variabilités climatiques dans les communes riveraines de la Réserve de Biosphère Transfrontalière du W (Bénin). Mémoire de DEA, FSA/UAC, 85 p.

14. Mbow M. A., Faye E.H., Kairé M., Akpo L.E., Diouf M., 2009Diversité d'une végétation ligneuse soudanienne dans les systèmes d'utilisation des terres du Sud-Ouest du Bassin arachidier du Sénégal. Journal des sciences et techniques, ISDN 0850-5802.

15. Saliou A.R., 2015. Modelisation predictive et cartographie de la dynamique des parcours de transhumance dans le contexte de variabilites climatiques dans le moyen-Bénin

16. Sounon Bouko B., Sinsin B. \& Bio Goura B., 2007. Effets de la dynamique d'occupation du sol sur la structure et la diversité floristique des forêts claires et savanes au Bénin. Tropicultura, 25 (4) : 193-199.

17. Von Maydell H.J., 1990. Arbres et arbustes du Sahel, leurs caractéristiques et leurs utilisations. GTZ. Marfrag. 150p. 
18. Wala K., 2004. La végétation de la chaine de l’Atakora au Benin : diversité floristique, phytosociologie et impact humain. Thèse de doctorat, Université de Lomé, 140 p.

19. Toko I., 2008. Etude de la variabilité spatiale de la biomasse herbacée, de la phénologie et de la structure de la végétation le long des topos séquences du bassin supérieur du fleuve Ouémé au Bénin. Thèse de Doctorat en Géographie, Université d'Abomey-Calavi, 241 p.

20. Tamou C., 2002. Etat des lieux quantitatifs et spatialisé de la transhumance dans la zone d'influence du Réserve de Biosphère Transfrontalière du $\mathrm{W}$ du fleuve Niger-Bénin. Thèse d'Ingénieur Agronome, FSA/UAC, $111 \mathrm{p}$.

21. Ouédraogo A., 2006. Diversité et dynamique de la végétation ligneuse dans la partie orientale du Burkina Faso. Thèse de doctorat, Université de Ouagadougou, Burkina Faso, 230 p.

22. Steinfeld H., Gerber P., Wassenaar T., Castel V., Rosales M., de Haan C., 2006. Livestock's long shadow, environmental issues and option. Rome: FAO, LEAD Initiative

23. Yossi, 1996. - Dynamique de la végétation post-culturale en zone soudanienne du Mali. Thèse doctorat. Option PopulationEnvironnement, Isfra, Bamako, Mali, 141 p. 